\title{
Major causes of organ and carcass condemnation and associated financial losses in cattle slaughtered at Kombolcha ELFORA abattoir from 2008-2012, Ethi- opia
}

\author{
Desie Sheferaw ${ }^{1 *}$ and Kedir Abdu ${ }^{2}$ \\ ${ }^{1}$ Hawassa University, School of Veterinary Medicine, Hawassa, Ethiopia \\ ${ }^{2}$ MoLFD, Amhara Regional State, \\ *Corresponding author: Hawassa University, School of Veterinary Medicine, Hawassa, Ethiopia. \\ Email: mereba480@gmail.com \\ https://dx.doi.org/10.4314/evj.v21i1.5
}

\begin{abstract}
The purpose of this study was to investigate the major causes of organ and/or carcass condemnation and to estimate the financial loss encountered in cattle slaughtered at ELFORA abattoir. The study is based on retrospective analysis of five year data record at slaughter house cattle $(n=21864)$ and active abattoir survey involving pre and postmortem inspection of 1200 cattle conducted from October 2013 to April 2014. From a total of 23,064 slaughtered cattle, both during retrospective and cross-sectional studies, 15,971 (69.2\%) had one or more than one type of abnormalities that lead to organ and/or carcass condemnation. Of these $67.7 \%, 40.3 \%, 38.8 \%, 23.0 \%, 19.9 \%, 7.0 \%$ and $5.0 \%$ were accounted to pathologies attributed to liver, kidney, lung, spleen, heart, tongue and heads condemnation, respectively. Liver was the most commonly condemned organ due to zoonotic parasites (32.8\%), which include Fasciola species, Cysticercus bovis and Cystic echinococcosis. Parasites were the major causes of organs particularly liver, lung and heart condemnation. Carcass condemnation was mainly attributed to TB and TB like lesions, oedema, abscess and Cysticercus bovis. Overall, the total economic loss incurred due to organ and carcass condemnation was estimated to reach 3,522,005.00 Ethiopian Birr during the study period. Hence, awareness creation through animal health extension work and treatment of sick animal will play key role in reducing the loss. Implementation of detailed meat inspection at the abattoir is recommended. Also further study to identify the causative agents of abscess; and to characterize hydatid cysts, and causes of tubercle like lesions will be important for designing control strategy.
\end{abstract}


Keywords: Carcass; Condemnation; ELFORA; Fasciolosis; Hydatid cyst; Organ; Ethiopia

\section{Introduction}

The Ethiopian livestock sector was contributing considerable portion to the economy of the country, and still it is promising in the economic development of the country. There are about 52.13 million heads of cattle in the country (CSA, 2012). However, their contribution is not yet fully exploited, because of losses that occur as a result of livestock diseases. At abattoir, organs and carcasses were condemned due to various pathogen and /or pathological conditions. Some of the pathogens are zoonotic and has public health importance; and all causes of condemnation incur serious economic losses to the livestock industry.

Abattoirs played an important role in surveillance of various zoonotic diseases, and it allows for all animals passing in to human food chain to be examined for unusual signs, lesions or specific disease (Alton et al., 2010). The purpose of meat inspection is to protect public health and to provide risk free products to the society. Also, it provides information that can be utilized for animal diseases control (Gracey et al., 1999). Abattoir data is an excellent option for detecting diseases of both economic and public health importance (Fufa Abunna et al., 2010; Arbabi et al., 2006), especially in ascertaining the extent to which human is exposed to certain zoonotic diseases in addition to estimating the financial implications of carcass condemnations (Yilma Jobre et al., 1996). Meat inspection assists to detect certain diseases of livestock and prevent the distribution of infected meat that could give rise to disease in animal and human being and to insure competitiveness of products in the local market (Hinton and Green, 1993; Vanlontestijn, 1993).

In the tropics, among the cattle diseases parasites are responsible for greater loss to meat industry than any other disease (Hinton and Green, 1993). Most studies undertaken in various abattoirs of the country (Yalew Tefera et al., 2016; Nebyou Moje et al., 2014; Alembrhan Assefa and Haylegebriel Tesfay, 2013; Fufa Abunna et al., 2013; Amene Fekadu et al., 2012; Genet Mesele et al., 2012; Fufa Abunna et al., 2012; Nurit Mohammed et al., 2012; Yifat Denbarga et al., 2011) were confined to a short period, limited number of data and most commonly on parasitic diseases such as fasciolosis, hydatidosis and Cysticercosis bovis. Therefore, the objectives of this study were to assess the major 
causes of organs and carcass condemnation over a period of time, to know the prevailing zoonotic diseases and to estimate the direct financial loss attributed to the condemned organs and carcasses of cattle slaughtered at Kombolcha ELFORA abattoir.

\section{Materials and Methods}

\section{Study area and animals}

The study was conducted at Kombolcha ELFORA export abattoir from October 2013 to April 2014. The abattoir is found in Kombolcha town, north east of Amhara Regional State, Ethiopia. The study animals were cattle brought from those districts around Kombolcha, and slaughtered in the abattoir.

\section{Study design and data collection}

For this study, both retrospective and active abattoir survey were used. The retrospective study involved analyzing recorded data from the abattoir record book on the causes of organ and carcass condemnation in the last five years, January 2008 to December 2012. All slaughtered animals were inspected by qualified meat inspector and all observed lesions were recorded on the record book of the abattoir. These data were used with the permission of the abattoir management.

The active abattoir survey was conducted from October 2013 to April 2014 using the regular meat inspection procedure to identify the major causes of organ and/or carcass condemnation. In this study each week three days visit was made for ante mortem and postmortem examination of slaughtered cattle. On each visit day, about 20 cattle were examined and the animals were selected by systematic random sampling techniques; and accordingly, a total of 1,200 cattle were examined.

\section{Method of examination}

The method employed for this study was routine meat inspection protocols of the former "Meat Inspection and Quarantine Division, MoA" (Solomon Hailemariam, 1975). During routine meat inspection, the disease conditions were grossly diagnosed based on pathological changes as described by Gracey et al (1999) and Herenda et al (1994). Cysts and adult parasites were identified 
at the spot, and if the lesions were doubtful samples were taken to Kombolcha regional veterinary laboratory for further examination and confirmation.

All animals slaughtered in the abattoir were rendered insensible to pain by stunning, humane slaughter, and the post mortem inspection was done following the ethical procedure described in "Meat Inspection Proclamation №. 274/1970" (Negarit Gazeta, 1970).

\section{Assessment of direct economic loss}

Total number of cattle slaughtered, average local market price during the study period and number of each condemned organ and carcass were used to estimate the financial loss. The total numbers of cattle slaughtered in the last five years were collected from the abattoir record book. The average retail price of each organ and carcass per kilogram was collected using open ended questionnaire from the butcheries in Kombolcha town.

The total economic loss encountered during both active and retrospective studies is computed by using the following:

Total loss $=\left(\mathrm{C}_{\mathrm{kg}} * \mathrm{P}_{\mathrm{f}}\right.$ per kg $)+\left(\mathrm{L} * \mathrm{P}_{\mathrm{L}}\right)+\left(\mathrm{Lu} * \mathrm{P}_{\mathrm{Lu}}\right)+\left(\mathrm{H} * \mathrm{P}_{\mathrm{H}}\right)+\left(\mathrm{K} * \mathrm{P}_{\mathrm{K}}\right)+(\mathrm{S}$ * $\left.\mathrm{P}_{\mathrm{S}}\right)+\left(\mathrm{H}_{\mathrm{e}} * \mathrm{P}_{\mathrm{He}}\right)+\left(\mathrm{T} * \mathrm{P}_{\mathrm{T}}\right)$
Where, $\mathrm{C}=$ Flesh in $\mathrm{kg}$,
$\mathrm{P}_{\mathrm{f}}=$ Price of carcass

in $\mathrm{kg}$

$\mathrm{L}=\mathrm{N} \underline{\mathrm{o}}$ of liver condemned

$\mathrm{Lu}=$ No of lung

$\mathrm{H}=\mathrm{No}$ of heart

$\mathrm{K}=$ No of kidney

$\mathrm{S}=$ No of spleen

$\mathrm{T}=$ No of tongue

$\mathrm{H}_{\mathrm{e}}=$ Head

$\mathrm{P}_{\mathrm{L}}=$ Price of liver

$\mathrm{P}_{\mathrm{Lu}}=$ Price of lung

$\mathrm{P}_{\mathrm{H}}=$ Price of heart

$\mathrm{P}_{\mathrm{K}}=$ Price of kidney

$\mathrm{P}_{\mathrm{S}}=$ Price of spleen

$\mathrm{P}_{\mathrm{T}}=$ Price of tongue

$\mathrm{P}_{\mathrm{He}}=$ Price of head

\section{Data management and analysis}

All collected data were entered in to Microsoft excel spread sheet and summarized by descriptive statistical methods like mean, percentage and proportion. 


\section{Results}

\section{General information}

From a total of 23,064 cattle slaughtered, both during retrospective and active studies, 15,971 (69.2\%) animals had one or more than one type of pathological conditions that lead to organ and/or carcass condemnation. Of these, 50.3\%, $35.6 \%, 33.7 \%, 20.0 \%, 15.7 \%, 5.0 \%$ and $2.5 \%$ were accounted to livers, lungs, kidneys, spleens, hearts, tongues and heads condemnation, respectively. Overall, parasitic (Cysticercus bovis, Cystic echinococcosis and Fasciola species) causes of condemnation accounted for $32.8 \%$ of liver condemnation. Fasciola species $(25.6 \%)$ was the major causes of liver condemnation (Table 1).

\section{Retrospective study}

In the retrospective study, between 2008 and 2012, the most commonly condemned organs were liver, lung and kidney with condemnation rate of $50.82 \%$, $36.4 \%$ and $35.33 \%$, respectively. Fasciola $(25.97 \%)$ was the major for liver condemnation, which was followed by hepatitis (14.27\%) and Cystic echinococcosis (3.53\%). The total number of animals slaughtered during the five years, retrospective study, and the number of various organs condemnation is shown in Table 2 . The abattoir record showed that about $72(0.33 \%)$ whole carcasses were condemned mainly due to TB and TB like lesions, oedema, abscess and Cysticercus bovis. 
Table 1. Overall causes and proportions of organs and carcasses condemned, Kombolcha ELFORA abattoir $(n=23,064)$

\begin{tabular}{|c|c|c|c|c|c|c|c|c|}
\hline $\begin{array}{l}\text { Cause of } \\
\text { Condemnation }\end{array}$ & Liver & Lung & Heart & Kidney & Spleen & Head & Tongue & Carcass \\
\hline Abscess & $\begin{array}{c}693 \\
(3.0 \%)\end{array}$ & $\begin{array}{l}214 \\
(0.9 \%)\end{array}$ & - & - & $\begin{array}{c}29 \\
(0.1 \%)\end{array}$ & $\begin{array}{c}385 \\
(1.7 \%)\end{array}$ & $\begin{array}{c}410 \\
(1.8 \%)\end{array}$ & - \\
\hline C. bovis & $\begin{array}{c}792 \\
(3.4 \%)\end{array}$ & - & $\begin{array}{c}7 \\
(0.03 \%)\end{array}$ & - & - & $51(0.2 \%)$ & $\begin{array}{c}203 \\
(0.9 \%)\end{array}$ & - \\
\hline Calcification & $\begin{array}{c}39 \\
(0.2 \%)\end{array}$ & - & - & - & - & - & - & - \\
\hline Cirrhosis & $\begin{array}{c}91 \\
(0.4 \%)\end{array}$ & - & - & - & - & - & - & - \\
\hline Cystic echinococcosis & $\begin{array}{c}855 \\
(3.7 \%)\end{array}$ & $\begin{array}{l}2215 \\
(9.6 \%)\end{array}$ & $\begin{array}{c}157 \\
(0.7 \%)\end{array}$ & $\begin{array}{l}145 \\
(0.6 \%)\end{array}$ & $\begin{array}{c}334 \\
(1.4 \%)\end{array}$ & - & - & - \\
\hline Fasciolosis & $\begin{array}{c}5915 \\
(25.6 \%)\end{array}$ & - & - & - & - & - & & \\
\hline Emphysema & - & $\begin{array}{l}2135 \\
(9.2 \%)\end{array}$ & - & - & - & - & - & - \\
\hline Oedema & - & $\begin{array}{c}1 \\
(0.004 \%)\end{array}$ & $\begin{array}{l}1238 \\
(5.4 \%)\end{array}$ & $\begin{array}{c}1836 \\
(8.0 \%)\end{array}$ & - & $\begin{array}{c}86 \\
(0.4 \%)\end{array}$ & $\begin{array}{c}293 \\
(1.3 \%)\end{array}$ & $8 \mathrm{WC}$ \\
\hline $\begin{array}{l}\text { Pneumonia and } \\
\text { CBPP }\end{array}$ & - & $\begin{array}{c}3531 \\
(15.3 \%)\end{array}$ & - & - & - & - & - & - \\
\hline $\begin{array}{l}\text { TB and TB like } \\
\text { lesions }\end{array}$ & $\begin{array}{c}60 \\
(0.3 \%)\end{array}$ & $\begin{array}{l}110 \\
(0.5 \%)\end{array}$ & $\begin{array}{c}47 \\
(0.2 \%)\end{array}$ & $\begin{array}{c}76 \\
(0.3 \%)\end{array}$ & $\begin{array}{c}46 \\
(0.2 \%)\end{array}$ & $\begin{array}{c}42 \\
(0.18 \%)\end{array}$ & $\begin{array}{c}44 \\
(0.19 \%)\end{array}$ & $60 \mathrm{WC}$ \\
\hline Pericarditis & - & - & $\begin{array}{c}1333 \\
(5.8 \%)\end{array}$ & - & - & - & - & - \\
\hline $\begin{array}{l}\text { Haemorrhage and/or } \\
\text { haematoma }\end{array}$ & $\begin{array}{c}16 \\
(0.07 \%)\end{array}$ & $\begin{array}{c}1 \\
(0.004 \%)\end{array}$ & $\begin{array}{c}655 \\
(2.84 \%)\end{array}$ & $\begin{array}{c}1993 \\
(8.64 \%)\end{array}$ & $\begin{array}{c}1288 \\
(5.58 \%)\end{array}$ & - & $\begin{array}{c}165 \\
(0.72 \%)\end{array}$ & - \\
\hline Hepatitis & $\begin{array}{c}3121 \\
(13.53 \%)\end{array}$ & - & - & - & - & - & - & - \\
\hline Nephritis & - & - & - & $\begin{array}{c}3340 \\
(14.5 \%)\end{array}$ & - & - & - & - \\
\hline Calculi & & & & $\begin{array}{c}375 \\
(1.6 \%)\end{array}$ & & & & \\
\hline Splenomegally & - & - & - & - & $\begin{array}{c}2919 \\
(12.7 \%)\end{array}$ & - & - & - \\
\hline Actinomycosis & - & - & - & - & - & $\begin{array}{c}5 \\
(0.02 \%)\end{array}$ & - & - \\
\hline Actinobacillosis & - & - & - & - & - & $\begin{array}{c}12 \\
(0.05 \%)\end{array}$ & - & - \\
\hline Bruising & - & - & - & - & - & - & - & $3 \mathrm{Kg}$ \\
\hline Tumor & $\begin{array}{c}13 \\
(0.06 \%)\end{array}$ & $\begin{array}{c}3 \\
(0.01 \%)\end{array}$ & $\begin{array}{c}190 \\
(0.8 \%)\end{array}$ & $\begin{array}{c}10 \\
(0.04 \%)\end{array}$ & $\begin{array}{c}1 \\
(0.004 \%)\end{array}$ & $\begin{array}{c}3 \\
(0.01 \%)\end{array}$ & $\begin{array}{c}8 \\
(0.03 \%)\end{array}$ & $2 \mathrm{WC}$ \\
\hline Ulcer & - & - & - & - & - & - & $\begin{array}{c}184 \\
(0.8 \%)\end{array}$ & \\
\hline Total & $\begin{array}{c}11595 \\
(50.3 \%)\end{array}$ & $\begin{array}{c}8210 \\
(35.6 \%)\end{array}$ & $\begin{array}{c}3627 \\
(15.7 \%)\end{array}$ & $\begin{array}{c}7775 \\
(33.7 \%)\end{array}$ & $\begin{array}{c}4617 \\
(20.0 \%)\end{array}$ & $\begin{array}{c}584 \\
(2.5 \%)\end{array}$ & $\begin{array}{l}1307 \\
(5.7 \%)\end{array}$ & $\begin{array}{c}70 \mathrm{WC}+ \\
3 \mathrm{Kg}\end{array}$ \\
\hline
\end{tabular}


Table 2. Causes and number of organs and carcasses $(\mathrm{kg})$ condemned from 2008 to $2012(n=21,864)$

\begin{tabular}{|c|c|c|c|c|c|c|c|c|}
\hline $\begin{array}{l}\text { Condemnation } \\
\text { cause }\end{array}$ & Liver & Lung & Heart & Kidney & Spleen & Head & Tongue & Carcass \\
\hline Abscess & $\begin{array}{c}661 \\
(3.02 \%)\end{array}$ & $\begin{array}{c}200 \\
(0.91 \%)\end{array}$ & $\begin{array}{c}37 \\
(0.17 \%)\end{array}$ & $\begin{array}{c}4 \\
(0.02 \%)\end{array}$ & $\begin{array}{c}25 \\
(0.11 \%)\end{array}$ & $\begin{array}{c}371 \\
(1.7 \%)\end{array}$ & $\begin{array}{c}391 \\
(1.79 \%)\end{array}$ & $\begin{array}{c}4 \mathrm{WC} \\
+5.75 \mathrm{~kg}\end{array}$ \\
\hline C. bovis & $\begin{array}{c}763 \\
(3.49 \%)\end{array}$ & - & $\begin{array}{c}246 \\
(1.13 \%)\end{array}$ & $\begin{array}{c}1 \\
(0.005 \%)\end{array}$ & $\begin{array}{c}1 \\
(0.005 \%)\end{array}$ & $\begin{array}{c}40 \\
(0.18 \%)\end{array}$ & $\begin{array}{c}169 \\
(0.77 \%)\end{array}$ & $3 \mathrm{WC}$ \\
\hline $\begin{array}{l}\text { Cystic } \\
\text { echinococcosis }\end{array}$ & $\begin{array}{c}772 \\
(3.53 \%)\end{array}$ & $\begin{array}{c}2092 \\
(9.57 \%)\end{array}$ & $\begin{array}{c}147 \\
(0.67 \%)\end{array}$ & $\begin{array}{c}137 \\
(0.63 \%)\end{array}$ & $\begin{array}{c}328 \\
(1.5 \%)\end{array}$ & - & - & - \\
\hline Fasciola & $\begin{array}{c}5679 \\
(25.97 \%)\end{array}$ & - & - & - & - & - & - & - \\
\hline Actinobacillosis & - & - & - & - & - & $\begin{array}{c}12 \\
(0.05 \%)\end{array}$ & - & - \\
\hline Actinomycosis & - & - & - & - & - & $\begin{array}{c}3 \\
(0.014 \%)\end{array}$ & - & - \\
\hline Bruising & - & - & - & - & - & $\begin{array}{c}293 \\
(1.34 \%)\end{array}$ & - & $2 \mathrm{~kg}$ \\
\hline Calculi & - & - & - & $\begin{array}{c}375 \\
(1.72 \%)\end{array}$ & - & - & - & - \\
\hline $\begin{array}{l}\text { Pneumonia and } \\
\text { CBPP }\end{array}$ & - & $\begin{array}{c}3464 \\
(15.84 \%)\end{array}$ & - & - & - & - & - & - \\
\hline Oedema & - & $\begin{array}{c}1 \\
(0.005 \%)\end{array}$ & $\begin{array}{c}1238 \\
(5.66 \%)\end{array}$ & $\begin{array}{c}1836 \\
(8.4 \%)\end{array}$ & - & $\begin{array}{c}86 \\
(0.39 \%)\end{array}$ & $\begin{array}{c}293 \\
(1.34 \%)\end{array}$ & $8 \mathrm{WC}$ \\
\hline Emphysema & - & $\begin{array}{c}2092 \\
(9.57 \%)\end{array}$ & - & - & - & - & - & - \\
\hline $\begin{array}{l}\text { Haemorrhage } \\
\text { and haematoma }\end{array}$ & $\begin{array}{c}16 \\
(0.07 \%)\end{array}$ & $\begin{array}{c}1 \\
(0.005 \%)\end{array}$ & $\begin{array}{c}655 \\
(3.0 \%)\end{array}$ & $\begin{array}{c}1993 \\
(9.12 \%)\end{array}$ & $\begin{array}{c}1288 \\
(5.89 \%)\end{array}$ & - & $\begin{array}{c}165 \\
(0.75 \%)\end{array}$ & - \\
\hline Hepatitis & $\begin{array}{c}3121 \\
(14.27 \%)\end{array}$ & - & - & - & - & - & - & - \\
\hline Nephritis & - & - & - & $\begin{array}{c}3288 \\
(15.04 \%)\end{array}$ & - & - & - & - \\
\hline Pericarditis & - & - & $\begin{array}{c}1274 \\
(5.83 \%)\end{array}$ & - & - & - & - & - \\
\hline TB & $\begin{array}{c}60 \\
(0.27 \%)\end{array}$ & $\begin{array}{c}106 \\
(0.48)\end{array}$ & $47(0.21 \%)$ & $\begin{array}{c}76 \\
(0.35 \%)\end{array}$ & $\begin{array}{c}46 \\
(0.21 \%)\end{array}$ & $\begin{array}{c}42 \\
(0.19 \%)\end{array}$ & $\begin{array}{c}44 \\
(0.2 \%)\end{array}$ & $55 \mathrm{WC}$ \\
\hline Tumor & $\begin{array}{c}13 \\
(0.06 \%)\end{array}$ & $\begin{array}{c}3 \\
(0.014 \%)\end{array}$ & $\begin{array}{c}190 \\
(0.87 \%)\end{array}$ & $\begin{array}{c}10 \\
(0.05 \%)\end{array}$ & $\begin{array}{c}1 \\
(0.005 \%)\end{array}$ & $\begin{array}{c}3 \\
(0.014 \%)\end{array}$ & $\begin{array}{c}8 \\
(0.037 \%)\end{array}$ & $2 \mathrm{WC}$ \\
\hline Ulcer & - & - & - & - & - & - & $\begin{array}{c}184 \\
(0.84 \%)\end{array}$ & - \\
\hline Splenitis & - & - & - & - & $\begin{array}{c}2893 \\
(12.23 \%)\end{array}$ & - & - & - \\
\hline Total & $\begin{array}{c}11113 \\
(50.83 \%)\end{array}$ & $\begin{array}{c}7959 \\
(36.4 \%)\end{array}$ & $\begin{array}{c}3834 \\
(17.54 \%)\end{array}$ & $\begin{array}{c}7724 \\
(35.33 \%)\end{array}$ & $\begin{array}{c}4582 \\
(20.96 \%)\end{array}$ & $\begin{array}{c}850 \\
(3.89 \%)\end{array}$ & $\begin{array}{c}1254 \\
(5.74 \%)\end{array}$ & $\begin{array}{c}72 \mathrm{WC} \\
+7.75 \mathrm{~kg}\end{array}$ \\
\hline
\end{tabular}

\section{Active abattoir survey}

From a total of 1200 cattle that were examined 510 (42.5\%) liver, 251 (20.9\%) lungs, 60(5\%) kidneys, 75(6.3\%) hearts, 36(3\%) spleens, 27(2.3\%) heads and $53(4.4 \%)$ tongues were condemned due to various causes. Parasites like Cysti- 
cercus bovis, Cystic echinococcosis and Fasciola species accounted for 29\% of liver condemnation. The detailed information for causes of condemnation was shown in Table 3.

Table 3. Causes and proportions of organs and carcasses condemned, at Kombolcha ELFORA during active survey $(n=1200)$

\begin{tabular}{|c|c|c|c|c|c|c|c|c|}
\hline $\begin{array}{l}\text { Condemnation } \\
\text { cause }\end{array}$ & Liver & Lung & Heart & Kidney & Spleen & Head & Tongue & Carcass \\
\hline Abscess & $\begin{array}{c}32 \\
(2.7 \%)\end{array}$ & $\begin{array}{c}14 \\
(1.2 \%)\end{array}$ & - & - & $\begin{array}{c}4 \\
(0.3 \%)\end{array}$ & $\begin{array}{c}14 \\
(1.2 \%)\end{array}$ & $\begin{array}{c}19 \\
(1.6 \%)\end{array}$ & - \\
\hline C. bovis & $\begin{array}{c}29 \\
(2.4 \%)\end{array}$ & - & $\begin{array}{c}6 \\
(0.5 \%)\end{array}$ & - & - & $\begin{array}{c}11 \\
(0.9 \%)\end{array}$ & $\begin{array}{c}34 \\
(2.8 \%)\end{array}$ & - \\
\hline Calcification & $\begin{array}{c}39 \\
(3.3 \%)\end{array}$ & - & - & - & - & - & - & - \\
\hline Cirrhosis & $\begin{array}{c}91 \\
(7.6 \%)\end{array}$ & - & - & - & - & - & - & - \\
\hline Cystic echinococcosis & $\begin{array}{c}83 \\
(6.9 \%)\end{array}$ & $\begin{array}{c}123 \\
(10.3 \%)\end{array}$ & $\begin{array}{c}10 \\
(0.8 \%)\end{array}$ & $\begin{array}{c}8 \\
(0.7 \%)\end{array}$ & $\begin{array}{c}6 \\
(0.5 \%)\end{array}$ & - & - & - \\
\hline Fasciolosis & $\begin{array}{c}236 \\
(19.7 \%)\end{array}$ & - & - & - & - & - & & \\
\hline Emphysema & - & $\begin{array}{c}43 \\
(3.6 \%)\end{array}$ & - & - & - & - & - & - \\
\hline Pneumonia & - & $\begin{array}{c}67 \\
(5.6 \%)\end{array}$ & - & - & - & - & - & - \\
\hline TB like lesions & - & $\begin{array}{c}4 \\
(0.3 \%)\end{array}$ & - & - & - & - & - & $\begin{array}{c}5 \\
(0.4 \%)\end{array}$ \\
\hline Pericarditis & - & - & $\begin{array}{c}59 \\
(4.9 \%)\end{array}$ & - & - & - & - & - \\
\hline Nephritis & - & - & - & $\begin{array}{c}15 \\
(1.3 \%)\end{array}$ & - & - & - & - \\
\hline Hydronephrosis & - & - & - & $\begin{array}{c}37 \\
(3.1 \%)\end{array}$ & - & - & - & - \\
\hline Splenomegally & - & - & - & - & $\begin{array}{c}26 \\
(2.2 \%)\end{array}$ & - & - & - \\
\hline Actinomycosis & - & - & - & - & - & $\begin{array}{c}2 \\
(0.2 \%)\end{array}$ & - & - \\
\hline Bruising & - & - & - & - & - & - & - & $\begin{array}{c}1 \\
(0.08 \%)\end{array}$ \\
\hline Total & $\begin{array}{c}510 \\
(42.5 \%)\end{array}$ & $\begin{array}{c}251 \\
(20.9 \%)\end{array}$ & $\begin{array}{c}75 \\
(6.3 \%)\end{array}$ & $\begin{array}{c}60 \\
(5 \%)\end{array}$ & $\begin{array}{c}36 \\
(3 \%)\end{array}$ & $\begin{array}{c}27 \\
(2.3 \%)\end{array}$ & $\begin{array}{c}53 \\
(4.4 \%)\end{array}$ & $\begin{array}{c}6 \\
(0.5 \%)\end{array}$ \\
\hline
\end{tabular}

\section{Economic loss}

The number of animals slaughtered from 2008 to 2012 and during active abattoir study, as well the number of organs and carcass condemned was shown in Table 4. The average price of liver, lung, kidney, heart, spleen, head, tongue and carcass/Kg were 90, 20, 20, 55, 5, 60, 40 and 140 Birr, respectively. The to- 
tal economic loss incurred both during retrospective study and active abattoir survey was 5,522,005.00 Ethiopian Birr.

Table 4. Number of organ and carcass $(\mathrm{kg})$ condemned during retrospective survey (2008 to 2012) and active abattoir study, and economic loss

\begin{tabular}{|c|c|c|c|c|c|c|c|c|}
\hline \multirow{2}{*}{$\begin{array}{l}\text { Slaughtered } \\
\text { and condemned }\end{array}$} & \multicolumn{5}{|c|}{ Retrospective study } & \multirow{2}{*}{$\begin{array}{l}\text { Active } \\
\text { survey }\end{array}$} & \multirow{2}{*}{ Total } & \multirow[b]{2}{*}{$\begin{array}{l}\text { Economic } \\
\text { loss (Birr) }\end{array}$} \\
\hline & 2008 & 2009 & 2010 & 2011 & 2012 & & & \\
\hline No slaughtered & 4229 & 5553 & 2851 & 5721 & 3510 & 1200 & 23064 & - \\
\hline Carcass (Kg) & 3300 & 4500 & 1800 & 2587.5 & 900 & 6 & 13093.5 & $1,833,090.00$ \\
\hline Liver & 2793 & 2243 & 1375 & 3321 & 1381 & 510 & 11623 & $1,046,070.00$ \\
\hline Lung & 1574 & 1926 & 1243 & 2109 & 1107 & 251 & 8210 & $164,200.00$ \\
\hline Heart & 887 & 787 & 738 & 898 & 524 & 75 & 3909 & $214,995.00$ \\
\hline Kidney & 1650 & 1901 & 1519 & 1804 & 850 & 60 & 7784 & $155,680.00$ \\
\hline Spleen & 949 & 895 & 773 & 1162 & 803 & 36 & 4618 & $23,090.00$ \\
\hline Head & 94 & 261 & 175 & 213 & 107 & 27 & 877 & $52,620.00$ \\
\hline Tongue & 211 & 391 & 340 & 235 & 77 & 53 & 1307 & $52,280.00$ \\
\hline $\begin{array}{l}\text { Economic loss } \\
\text { (Birr) }\end{array}$ & 845,460 & 987,470 & 499,525 & 816,780 & 311,765 & 61,005 & - & $3,522,005.00$ \\
\hline
\end{tabular}

\section{Discussion}

The current study revealed that Cysticercus bovis, Cystic echinococcosis and Fasciola species, and bovine tuberculosis were the most important causes of organ or carcass condemnation in slaughtered cattle at ELFORA abattoir. This study has revealed that a number of conditions result in the condemnation of organs and carcasses, and thus have great financial implications. From a total of 23,064 cattle slaughtered 15,971 (69.2\%) animals had one or more than one type of abnormalities that lead to organ and/or carcass condemnation. Of these $50.3 \%, 35.6 \%, 33.7 \%, 20 \%, 15.7 \%, 5.0 \%$ and $2.5 \%$ were accounted to livers, lungs, kidneys, spleens, hearts, tongues and heads condemnation, respectively. Liver was the most commonly condemned organ primarily due to parasites (32.8\%), which include Fasciola species, Cysticercus bovis and Cystic echinococcosis. Among the parasitic causes, fasciolosis accounted for 25.6\% overall liver condemnation, and $19.7 \%$ and $26.0 \%$ of liver condemnation during active abattoir survey and retrospective studies, respectively (Table 1 and 3). Fasciola species was reported to be the major cause of liver condemnation in Ethiopia (Fufa Abunna and Debele Hordofa, 2013; Alembrhan Assefa and Haylegebriel Tesfaye, 2013; Edo et al., 2014; Genet Mesele et al., 2012; Yalew Tefera et al., 2016), and other parts of Africa (Alawa et al., 2011; Tembo and 
Nonga, 2015). The other causes for liver condemnation in order of abundance were hepatitis (14.27\%), Cystic echinococcosis (3.7\%), Cysticercus bovis (3.4\%) and abscess (3.0\%) (Table 1). This result is in line with the report of Alembrhan Assefa and Haylegebriel Tesfay (2013) and Genet Mesele et al. (2012). Both Cystic echinococcosis (Fufa Abunna and Debele Hordofa, 2013; Abebe Fromisa and Yilma Jobre, 2012) and Cysticercus bovis (Kumar and Gebrehiwot Tadesse, 2011) were widespread and endemic parasitic disease in most parts of Ethiopia; and causing considerable direct and indirect economic loss from organ condemnation, decreased carcass weight and reduced milk yield.

Lung was the second most commonly condemned organ (35.6\%). Lung was condemned due to pneumonia (15.3\%), Cystic echinococcosis (9.6\%), emphysema (9.3\%), abscess (0.9\%), and TB and TB like lesions (0.5\%). Similarly, pneumonia, hydatid cyst and emphysema were reported from various abattoirs in the country as major and top causes for lung condemnation (Alembrhan Assefa and Haylegebriel Tesfay, 2013; Nebyou Moje et al., 2014; Yifat Denbarga et al., 2011). Kidney was the third most commonly condemned organ (33.6\%), which was mainly due to nephritis, haemorrhage, oedema and hydronephrosis (Table 1). The forth most commonly condemned organ was spleen (20.0\%), which was condemned mainly due to haematoma and hydatid cyst. Then, it was followed by heart $(15.7 \%)$, which was condemned primarily due to pericarditis $(5.8 \%)$, oedema (5.4\%), haemorrhage (2.8\%), hydatid cyst (0.7\%) and Cysticercus bovis (1.1\%). Different authors reported that these abnormalities were known to cause the condemnation of heart in various abattoirs in Ethiopia (Amene Fekadu et al., 2012; Genet Mesele et al., 2012; Nebyou Moje et al., 2014; Yifat Denbarga et al., 2011).

During both the retrospective study and active abattoir survey a total of 74 cattle whole carcasses were condemned due to tuberculosis, generalized oedema, abscess, Cysticercus bovis and unidentified tumors. These conditions were known to be major causes for the condemnation of carcass in many parts of Ethiopia (Genet Mesele et al., 2012; Nebyou Moje et al., 2014). Carcass condemnation due to tuberculosis and/or tubercul like lesion could be more than what had been recorded in this study. Because routine meat inspection has limitation in detecting bovine tuberculosis (Demelash Biffa et al., 2010 and Mihreteab Bekele and Indris Belay, 2011). Abscess, Cysticercus bovis, oedema, tuberculosis and tubercle like lesion were the major causes for tongue, head and carcass condemnation. These conditions were reported as the common 
cause of condemnation either partly or totally for these organs (Fufa Abunna and Debele Hordofa, 2013; Genet Mesele et al., 2012; Nebyou Moje et al., 2014).

Overall the total economic loss incurred due to organ and carcass condemnation was 3,522,005.00 Ethiopian Birr. This loss could be much higher if the indirect loss is computed and incorporated.

In conclusion liver, kidney, lung, spleen and heart were the most commonly condemned organs. The major causes of their partial or total condemnation were Fasciola species, Cystic echinococcosis, pneumonia, oedema, nephritis and hepatitis. But the major causes of carcass condemnation were TB and TB like lesions, oedema, abscess and Cysticercus bovis. So, these diseases and/or pathological conditions were resulting in considerable financial loss in Kombolcha ELFORA abattoir. Hence, animal health extension work should be in place to create awareness of animal producers on issues like proper disposal of condemned organs and/or carcass and treatment of sick animals. Moreover, strengthening meat inspection, detailed meat inspection, at the abattoir is recommended. Further study is mandatory to identify the causative agents of abscess, to characterize Cystic echinococcosis, which can play key role in the control of hydatidosis.

\section{Acknowledgements}

The authors are grateful to Dr Hareguwa, meat inspector at Kombolcha ELFORA abattoir, for her great help and encouragement during the study period. We would like also to appreciate the help of other meat inspectors and staff of Kombolcha ELFORA abattoir.

\section{References}

Abunna, F. and Hordofa, D., 2013. Major causes of organ condemnation for cattle and its financial impact at Wolaita Sodo municipality abattoir, Southern Ethiopia. Global Vet., 11(6), 730-734

Abunna, A., Fentaye, S., Megersa, B. and Regassa, A. 2012. Prevalence of bovine hydatidosis in Kombolcha ELFORA abattoir, North Eastern Ethiopia. Open J. Anim. Sci., 2(4), 281-286 
Abunna, F., Asfaw, L., Megersa, B.and Regassa, A., 2010. Bovine fasciolosis: Coprological, abattoir survey and its economic impact due to liver condemnation at Sodo Municipal abattoir, Southern Ethiopia. Trop. Anim. Hlth. Prod., 42, 289-292

Alawa, C.B.I., Etukudo-Joseph I. and Alawa, J.N., 2011. A 6-year survey of pathological conditions of slaughtered animals at Zango abattoir in Zaria, Kaduna State, Nigeria. Trop. Anim. Hlth. Prod., 43, 127-131

Alton, G.D., Lpeah, D., Bateman, K.J., McNab, W.B. and Berk, O., 2010. Factors associated with whole condemnation rates in provincially inspected abattoir in Ontario 2001-2007: Implication for food animal syndromic surveillance. BMC Vet. Res., 6, 42.

Amene, F., Eskindir, L.and Dawit, T., 2012. Cause, Rate and Economic Implication of Organ Condemnation of Cattle Slaughtered at Jimma Municipal Abattoir, Southwestern Ethiopia. Global Vet., 9(4), 396-400.

Arbabi, M. and Hooshyar, H., 2006. Survey of Echinococcosis and Hydatidosis in Kashun Region, Central Iran. Ir. J. Pub. Hlth., 35, 75-81.

Assefa, A. and Tesfay, H., 2013. Major causes of organ condemnation and economic loss in cattle slaughtered at Adigrat municipal abattoir, northern Ethiopia. Vet. World, 6(10), 734-738

Bekele, M. and Belay, I., 2011. Evaluation of Routine Meat Inspection Procedure to Detect Bovine Tuberculosis Suggestive Lesions in Jimma Municipal Abattoir, South West Ethiopia. Global Vet., 6(2), 172-179

Biffa, D., Bogale, A. and Skjerve, E., 2010. Diagnostic efficiency of abattoir meat inspection service in Ethiopia to detect carcasses infected with Mycobacterium bovis: Implications for public health. BMC Pub. Hlth., 10, 462.

CSA, 2012. Agricultural sample survey Volume II, Report on livestock and livestock characteristics, Central Statistic Authority, Addis Ababa, Ethiopia. Pp. 35-49

Edo, J.J., Pal, M.and Rahman, M.T., 2014. Investigation into major causes of organs condemnation in Bovine slaughtered at Adama municipal abattoir and their economic importance. Haryana Vet., 53(2), 139-143

Fromsa, A. and Jobre, Y., 2012. Estimated annual economic loss from organ condemnation, decreased carcass weight and milk yield due to bovine hydatidosis (Echinococcus granulosus, Batsch, 1786) in Ethiopia. Ethiop. Vet. J., 16(2), 1-14

Gracey, J.F., Collins, D.S. and Huey, R.J., 1999. Meat hygiene, $10^{\text {th }}$ Edition, London. W. B. Sounders Company Ltd. Pp. 758.

Hailemariam, S., 1975. A brief analysis of the activities of the meat inspection and quarantine division, Ministry of Agriculture, Addis Ababa, Ethiopia. 
Herenda, D., Chambers, P.G., Ettriqui, A., Seneviratne P. and da Silva, T.J.P., 1994. Manual of meat inspection for developing countries. Animal Production and Health Paper 119, FAO, Rome, Italy. Pp. 335

Hinton, M. and Green, L., 1993. Meat inspection which goes though University of Bristol, Langford, UK. Vet. J.,15(2), 91-92.

Jobre, Y., Lobago, F., Tiruneh, R., Abebe, G. and Dorchies, P.H., 1996. Hydatidosis in three selected regions of Ethiopia: An assessment trial on the prevalence, economic and public health importance. Rev. Med. Vet., 147, 797-804.

Kumar, A. and Tadesse, G., 2011. Bovine cysticercosis in Ethiopia: a review. Ethiop. Vet. J., 5(1), 15-35

Mesele, G., Guadu, T., Bogale, B. and Chanie, M., 2012. Pathological Conditions Causing Organ and Carcass Condemnation and Their Financial Losses in Cattle Slaughtered in Gondar, Northwest Ethiopia. Afr. J. Basic. Appl. Sci., 4(6), 200-208.

Mohammed, N., Hailemariam Z. and Mindaye, S. (2012): Major Cause of Liver Condemnation and Associated Financial Loss at Kombolcha Elfora Abattoir, South Wollo, Ethiopia. Eur. J. Appl.Sci., 4 (4), 140-145

Moje, N., Abdeta, D., Kebede, S., Terfa, T., Desissa, F. and Regassa, A., 2014. Major Causes of Organs and Carcass Condemnation in Cattle Slaughtered at Nekemte Municipality Abattoir, East Wollega, Ethiopia. Global Vet., 13(3), 278-284

Negarit Gazeta, 1970: Meat inspection proclamation, №. 274/1970, Negarit Gazeta, $29^{\text {th }}$ year, №. 15 .

Tefera, Y., Mesfin, Z. and Muleta, W., 2016. Major causes and abnormalities of organ condemnation and financial loss in cattle slaughtered at Dessie municipal abattior South Eastern Ethiopia. J. Vet. Med. Anim. Hlth., 8(7), 56-63

Tembo, W. and Nonga, H.E., 2015. A survey of the causes of cattle organs and/or carcass condemnation, financial losses and magnitude of foetal wastage at an abattoir in Dodoma, Tanzania, Onderstepoort J. Vet. Res., 82(1), Art. \#855, 7 pages. http:// dx.doi. org/10.4102/ojvr.v82i1.855

Vanlontestijn, J.G., 1993. Integrated quality. Meat safety: A new approach. Meats Focus Int., 2, 123-128.

Yifat, D., Gedefaw D. and Desie, S., 2011. Major Causes of Organ Condemnation and Financial Significance of Cattle Slaughtered at Gondar ELFORA Abattoir, Northern Ethiopia. Global Vet.,7, 487-490. 